\title{
Inhibition of stimulated Raman scattering due to the excitation of stimulated Brillouin scattering
}

\author{
Yao Zhao,,$^{1,2}$ Lu-Le Yu, ${ }^{1,2}$ Su-Ming Weng, ${ }^{1,2}$ Chuang Ren, ${ }^{3,4}$ Chuan-Sheng Liu, ${ }^{5,6}$ and Zheng-Ming \\ Sheng ${ }^{1,2,7, \text { a) }}$ \\ ${ }^{1)}$ Key Laboratory for Laser Plasmas (MoE), School of Physics and Astronomy, Shanghai Jiao Tong University, Shanghai 200240, \\ China \\ ${ }^{2)}$ Collaborative Innovation Center of IFSA (CICIFSA), Shanghai Jiao Tong University, Shanghai 200240, \\ China \\ ${ }^{3)}$ Department of Mechanical Engineering and Laboratory for Laser Energetics, University of Rochester, Rochester, \\ New York 14627, USA \\ ${ }^{4)}$ Department of Physics and Astronomy, University of Rochester, Rochester, New York 14627, \\ USA \\ ${ }^{5)}$ East-West Space Science Center, University of Maryland, College Park, Maryland 20742, \\ USA \\ ${ }^{6}$ Chao Kuang Piu College, University of Macau, Macau 999078, China \\ ${ }^{7)}$ SUPA, Department of Physics, University of Strathclyde, Glasgow G4 ONG, UK
}

The nonlinear coupling between stimulated Raman scattering (SRS) and stimulated Brillouin scattering (SBS) of intense laser in underdense plasma is studied theoretically and numerically. Based upon the fluid model, their coupling equations are derived and a threshold condition of plasma density perturbations due to SBS for the inhibition of SRS is given. Particle-in-cell simulations show that this condition can be achieved easily by SBS in the so-called fluid regime with $k_{L} \lambda_{D}<0.15$, where $k_{L}$ is the Langmuir wave number and $\lambda_{D}$ is the Debye length [Kline et al., 2006 Phys. Plasmas 13, 055906]. SBS can reduce the saturation level of SRS and the temperature of electrons in both homogeneous and inhomogeneous plasma. Numerical simulations also show that this reduced SRS saturation is retained even if the fluid regime condition mentioned above is violated at later time due to plasma heating.

\section{INTRODUCTION}

Stimulated Raman scattering (SRS) and stimulated Brillouin scattering (SBS) may cause significant laser energy loss and preheating of fusion targets ${ }^{1-5}$, which represent key issues in laser-driven inertial confinement fusion. For SRS, the incident laser decays into a scattered light plus an electron plasma wave with frequency $\omega_{p e}$, and for SBS, the incident laser decays into a scattered light plus an ion acoustic wave with frequency $\omega_{i}$. The nonlinear evolution and saturation of SRS and SBS have been studied widely in the past decade. For example, the anomalously hot electrons due to rescattering of $\mathrm{SRS}^{5}$, trapped-particle instabilities ${ }^{6-8}$, and saturation of SRS by nonlinear frequency shifts ${ }^{9,10}$, and electron plasma wave wavefront bowing and self-focusing ${ }^{11-13}$. Most of these nonlinear mechanisms are developed in the kinetic regime $^{14}$, where $k_{L} \lambda_{D} \gtrsim 0.29$, with $k_{L}$ is the Langmuir wave number and $\lambda_{D}$ is the Debye length. Moreover, these mechanisms are often coupled to each other. SBS can be saturated by nonlinear frequency shifts ${ }^{15}$, ion trapping ${ }^{16}$ and ion acoustic decay ${ }^{17}$. Several methods have been proposed to reduce the growth of SRS, such as increasing laser bandwidths ${ }^{18-20}$, laser smoothing technique (e.g., smoothing by spectral dispersion, induced spatial incoherence and polarization smoothing $)^{21-27}$, and high initial plasma temperatures ${ }^{28}$.

Although the frequency of an ion acoustic wave is usually much lower than an electron plasma wave, the ion motions have important effects on the long time evolution of electro-

a)zmsheng@sjtu.edu.cn magnetic and Langmuir waves via the plasma density modulation. Theoretically this can be described by the well-known Zakharov equation ${ }^{29,30}$, which is related to plenty of nonlinear phenomena, such as Langmuir collapse and Langmuir turbulence, electromagnetic solitons, nonlinear evolution of the modulational instability, and spatiotemporal chaos ${ }^{31-34}$. Actually, a lot of theoretical work studied on the saturation of SRS due to Langmuir decay instabilities (LDI) ${ }^{32,35}$. However, this mechanism corresponds to an initially low ion acoustic wave (IAW) and is not associated with the experiment of a high-amplitude IAW driven by the strong SBS instability ${ }^{36}$. Meanwhile, the second decay instabilities obtain the energy from SRS, therefore SRS can not be totally suppressed. Recently, absolute SRS was shown to seed SBS via LDI in the context of shock ignition ${ }^{37}$. Some experiments had shown the inhibition of the electron plasma waves by the IAW, and the inhibition effects will be stronger by higher the level of the IAW. Under certain conditions, SRS disappears coincidentally with the onset of SBS or a seeding of an IAW ${ }^{38-41}$.

In this paper, we examine explicitly the inhibition of SRS due to the excitation of SBS in the fluid regime ${ }^{14,42}$ with $k_{L} \lambda_{D} \lesssim 0.15$, where a strong SBS can develop large amplitude ion density perturbations. A set of generalized coupling equations are derived, which can be reduced to the equations proposed in the previous work ${ }^{29,32,43}$. With these equations, we obtain a threshold of low frequency plasma density perturbations, beyond which SRS instability mode transforms into a harmonic mode. Particle-in-cell (PIC) simulations are carried out, which are coincident with the previous experiments. 


\section{NONLINEAR COUPLING OF SRS AND SBS}

Since the frequency for scattered light of SBS approximately equals the frequency of incident laser $\omega_{0}$, one can write $A_{t}=\left[A_{0}(x, t) e^{-i \omega_{0} t}+\right.$ c.c. $] / 2+\left[A_{1}(x, t) e^{-i \omega_{1} t}+\right.$ c.c. $] / 2$, $E_{x}=\left[E_{L}(x, t) e^{-i \omega_{L} t}+c . c.\right] / 2$, where $A_{0}$ is the sum temporal envelope of the incident laser and the SBS light wave, $A_{1}$ is the temporal envelope of stimulated Raman scattering light, and $E_{L}$ is the temporal envelope of the related Langmuir wave. Here we consider the envelopes varying slowly with respect to time, then $\partial_{t t}$ for the envelope profile can be ignored. For such a system, we can introduce the plasma electron density $n_{e}=n_{e}^{0}\left(1+n_{e}^{s}+n_{e}^{h}\right)$, where $n_{e}^{0}=m_{e} \omega_{p e}^{2} / 4 \pi e^{2}$ is the unperturbed plasma electron density, $n_{e}^{s}$ is the density perturbation on the ion-acoustic time scale, and $n_{e}^{h}$ corresponds to electron plasma wave oscillations. By using the equations for electromagnetic waves and electrostatic waves in plasma, one can obtain a set of coupled equations for $A_{0}, A_{1}$, and $E_{L}{ }^{32}$ :

$$
\begin{aligned}
& {\left[-2 i \omega_{0}\left(\partial_{t}+\nu_{0}\right)+\left(\omega_{p e}^{2}-\omega_{0}^{2}\right)-c^{2} \partial_{x x}\right] A_{0}=} \\
& -\omega_{p e}^{2} n_{e}^{s} A_{0}+\frac{e A_{1}}{m_{e}} \partial_{x} E_{L}, \\
& {\left[-2 i \omega_{1}\left(\partial_{t}+\nu_{1}\right)+\left(\omega_{p e}^{2}-\omega_{1}^{2}\right)-c^{2} \partial_{x x}\right] A_{1}=} \\
& -\omega_{p e}^{2} n_{e}^{s} A_{1}+\frac{e A_{0}}{m_{e}} \partial_{x} E_{L}^{*}, \\
& {\left[-2 i \omega_{L}\left(\partial_{t}+\nu_{e}\right)+\left(\omega_{p e}^{2}-\omega_{L}^{2}\right)-3 v_{t h}^{2} \partial_{x x}\right] E_{L}=} \\
& -\omega_{p e}^{2} n_{e}^{s} E_{L}-\frac{e \omega_{p e}^{2}}{2 m_{e} c^{2}} \partial_{x}\left(A_{0} A_{1}^{*}\right),
\end{aligned}
$$

where $e, m_{e}, v_{t h}, \nu_{0}, \nu_{1}$ and $\nu_{e}$ are the electron charge, electron mass, electron thermal velocity, and damping rates for the incident light, scattered light, and Langmuir wave, respectively. If SBS effects are not included, i.e., $n_{e}^{s}=0$, Eqs. (1)-(3) describe the SRS instabilities.

Separating the electron velocity and electric fields into two components at high and low frequencies, respectively, and using the equation of motion for ions, we can obtain the equation for the low frequency density fluctuation $n_{e}^{s}$ as

$$
\begin{aligned}
& \left(\partial_{t t}+2 \nu_{i} \partial_{t}-c_{s}^{2} \partial_{x x}\right) n_{e}^{s}= \\
& \frac{\omega_{p e}^{2}}{8 \pi m_{i} c^{2}} \partial_{x x}\left(\left|A_{0}\right|^{2}+\left|A_{1}\right|^{2}\right)+\frac{1}{16 \pi m_{i}} \partial_{x x}\left|E_{L}\right|^{2},
\end{aligned}
$$

where $m_{i}, c_{s}^{2}=\left(3 v_{i}^{2}+v_{t h}^{2}\right) / m_{i}$, and $\nu_{i}$ are the ion mass, ion acoustic velocity, and damping rate for the IAW, respectively.

Equations (1)-(4) describe the nonlinear coupling of SRS and SBS, they can be reduced to the form of Zakharov equations $^{29}$. Neglecting the SBS effects, we write $A_{0}=$ $A_{i} \exp \left(i k_{0} x\right), A_{1}=A_{s} \exp \left(i k_{1} x\right)$, and $E_{L}=E_{l} \exp \left(i k_{L} x\right)$, where $A_{i}, A_{s}$ and $E_{l}$ are the amplitudes of the incident laser, SRS scattering light and Langmuir wave, respectively. Assuming these amplitudes to vary slowly in space, they are reduced to the three wave coupling equations which describe $\mathrm{SRS}^{44}$
It is difficult to find analytical solutions to the coupling equations (1)-(4), which have been solved numerically in some reduced cases ${ }^{32,43}$. Generally, it indicates that the ion density perturbations can saturate SRS and modulate its scattering spectrum $^{45}$. Here we consider the absolute growth of SRS with slowly varying envelopes of $A_{1}$ and $E_{L}$. When the IAW is excited at the beginning, the back scattered light of SBS can be ignored, and the amplitude $A_{i}$ is a constant. Therefore, Eqs. (1)-(4) are reduced to

$$
\begin{gathered}
{\left[\frac{\partial}{\partial t}+\nu_{1}+i \frac{\omega_{p e}^{2} n_{e}^{s}}{2 \omega_{1}}\right] A_{s}-\frac{e k_{L}}{4 m_{e} \omega_{1}} A_{i} E_{l}^{*}=0,} \\
{\left[\frac{\partial}{\partial t}+\nu_{e}+i \frac{\omega_{p e}^{2} n_{e}^{s}}{2 \omega_{L}}\right] E_{l}-\frac{e k_{L} \omega_{p e}^{2}}{4 m_{e} c^{2} \omega_{L}} A_{s}^{*} A_{i}=0,}
\end{gathered}
$$

and $\omega_{i} \approx 0$ for a quasi-static ion density perturbation $n_{e}^{s}$.

By use of Eqs. (5) and (6) and only considering the damping for $E_{l}$, then we obtain the temporal evolution equation for the Langmuir wave

$$
\left[\partial_{t t}-i \delta \partial_{t}-\Gamma^{2}+\frac{\omega_{p e}^{4}\left|n_{e}^{s}\right|^{2}}{4 \omega_{1} \omega_{L}}-\frac{\omega_{p e}^{2}\left|n_{e}^{s}\right| \nu_{e}}{2 \omega_{1}}\right] E_{l}=0
$$

where $\delta=\omega_{p e}^{2}\left|n_{e}^{s}\right|\left(\omega_{L}-\omega_{1}\right) / 2 \omega_{1} \omega_{L}+i \nu_{e}, \Gamma=$ $k_{L} c \omega_{p e} a_{0} / 4 \sqrt{\omega_{1} \omega_{L}}$ is the linear growth rate for SRS without ion density modulations ${ }^{9}$, and $a_{0}=e A_{i} / m_{e} c^{2}$ is the normalized incident laser field. For the case without ion density perturbation and Langmuir wave damping, we have $E_{l} \sim \exp (\Gamma t)$. Equation (7) indicates that $E_{l}$ tends to be saturated when the envelope of ion density perturbation satisfies $\left|n_{e}^{s}\right|^{2} \omega_{p e}^{2}-2\left|n_{e}^{s}\right| \nu_{e} \omega_{L}-4 \omega_{1} \omega_{L} \Gamma^{2} / \omega_{p e}^{2} \gtrsim 0$. Letting $\beta=\nu_{e} \sqrt{\omega_{L} / \omega_{1}} / 2 \Gamma$, then the SRS instability mode transforms into a harmonic mode when the low frequency plasma density perturbation satisfies

$$
\left|n_{e}^{s}\right| \gtrsim k_{L} c\left(\sqrt{1+\beta^{2}}+\beta\right) a_{0} / 2 \omega_{p e}
$$

This density threshold is proportional to the incident laser amplitude $a_{0}$, implying that higher laser intensity may lead to higher saturation levels for both SRS and SBS. Note that the IAW due to SBS usually saturates at a low level in the case with strong electron kinetic effects ${ }^{46,47}$. This means that SBS may have a weak effect on SRS saturation in this regime. However, in the fluid regime ${ }^{14,42}$ where $k_{L} \lambda_{D} \lesssim 0.15$ with a high ratio of $^{48} Z T_{e} / T_{i}$, a strong SBS can develop with a large amplitude of ion density perturbations, satisfying the threshold Eq. (8). In the next section, we will use the PIC simulations to demonstrate that SRS inhibition does exist when the development of SBS increases to a certain level. 

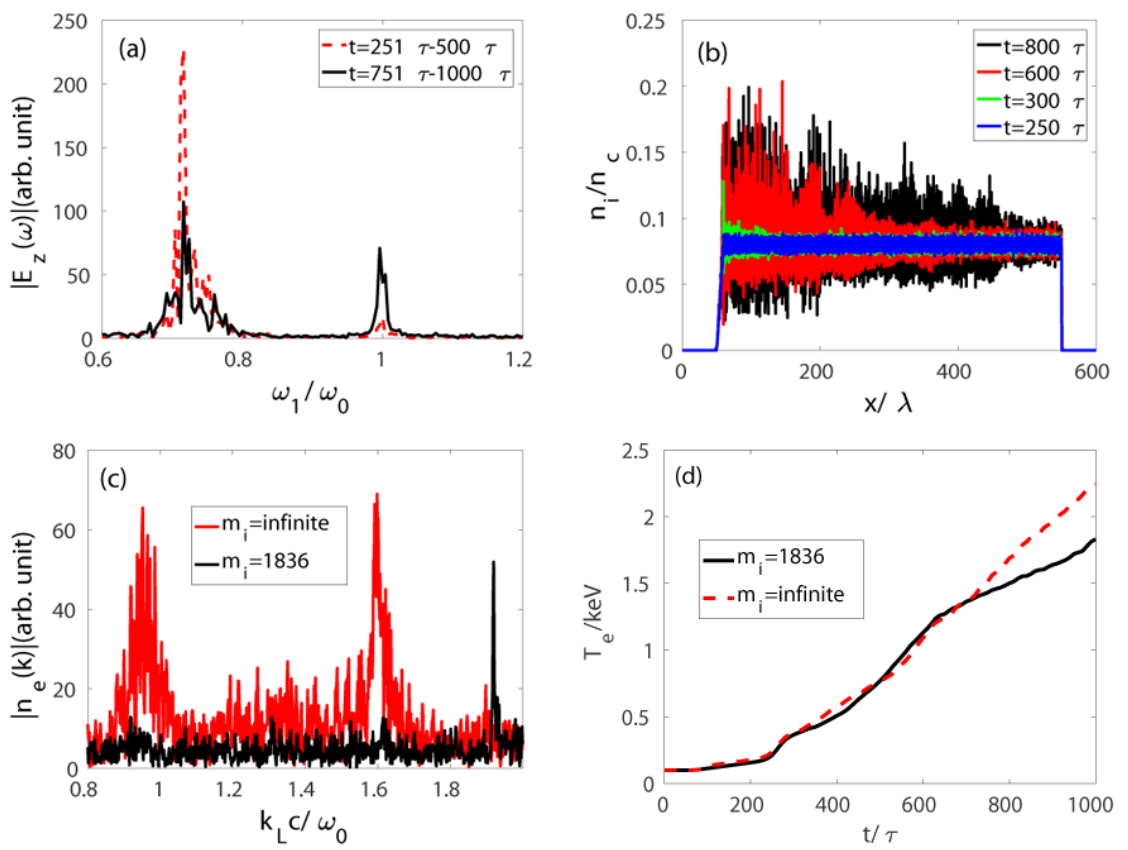

FIG. 1. Simulation results for $k_{L} \lambda_{D}=0.08$ and $Z T_{e} / T_{i} \gg 10$ with laser amplitude $a_{0}=0.05$. (a) and (b) ion mass is $m_{i}=1836$. (a) Snapshots of Fourier spectra of the backscattered light at different periods. (b) Snapshots of spatial distributions of the ion density at different time. (c) and (d) ion mass is infinite. (c) Snapshots of Fourier spectra of the electron density perturbations at $t=900 \tau$ with different ion masses. (d) Temporal evolution of the electron temperature with different ion masses.

\section{ONE-DIMENSIONAL PIC SIMULATIONS OF SRS SATURATION IN HOMOGENEOUS PLASMA}

PIC simulations using the code $\mathrm{KLAP}^{49}$ have been carried out to investigate the interactions between SRS and SBS. Onedimensional (1D) simulations are performed first to study the possible suppression of SRS due to SBS in homogeneous plasma. The length of the simulation box is $600 \lambda$, where the plasma occupies a region from $50 \lambda$ to $550 \lambda$ with $\lambda$ the incident laser wavelength in vacuum. We put 100 cells per wavelength and 50 particles per cell. The homogeneous plasma density is $n_{e}=0.08 n_{c}$ with a linear density ramp of $10 \lambda$ in the front, where $n_{c}$ is the critical electron density for the incident laser. The initial plasma temperature is $T_{e}=100 \mathrm{eV}$ and $T_{i}=0.8 \mathrm{eV}$, which corresponds to $k_{L} \lambda_{D}=0.08$ and $Z T_{e} / T_{i} \gg 10$, i.e., in the fluid regime. The charge of ion$\mathrm{s}$, which is normalized by electron charge, is $Z=1$. To study the effects of SBS on SRS, the ion mass is taken to be $m_{i}=1836$ or infinite (i.e., with immobile ions), which are normalized by electron mass. Note that the linear growth rate of Brillouin backscattering ${ }^{9,28} \Gamma_{B B S_{\perp}} \propto 1 / \sqrt{m_{i}}$, indicating that with the increase of ion mass, the growth rate of SBS is decreased. For infinite weights of ions, there is no SBS developed. A linearly-polarized semi-infinite pump laser with a $25 \lambda$ rising edge in the front incidents from the left boundary of the simulation box with a uniform amplitude $a_{0}=0.05$. The relation between the laser intensity $I_{0}$ and $a_{0}$ is given by $a_{0}=\sqrt{I_{0}\left(\mathrm{~W} / \mathrm{cm}^{2}\right)[\lambda(\mu \mathrm{m})]^{2} / 1.37 \times 10^{18}}$. The space and time given in the following are normalized by the laser wavelength in vacuum $\lambda$ and the laser period $\tau$.
We diagnose the backscattered light at $x=30 \lambda$ and plot its Fourier spectra at different periods with $m_{i}=1836$ in Fig. 1(a). The frequencies of backscattered light for SRS and SB$\mathrm{S}$ are found at $\omega_{1} \approx 0.72$ and $\omega_{1} \approx 1$, respectively ${ }^{28}$. This figure shows the SRS signal is lower during [750 $\tau, 1000 \tau]$ than during $[250 \tau, 500 \tau]$, suggesting that the SRS backscattered light tends to be reduced after certain time. Now let us examine the ion density perturbation. According to Eq (8), we can estimate the threshold for SRS saturation to be $\left|n_{e}^{s}\right| n_{e}^{0} \gtrsim 0.014 n_{c}$ for our simulation parameters. When considering the collisionless plasma wave damping effects, for example taking $\beta=0.25$, i.e., $\nu_{e}=0.01 \omega_{0},\left|n_{e}^{s}\right| n_{e}^{0}$ is slightly increased. It is expected that $\left|n_{e}^{s}\right| \simeq Z\left|\delta n_{i}\right|$ at least in the linear regime, where $\delta n_{i}$ is the ion density perturbation. At $t=250 \tau$, the ion density does not show obvious perturbations, as shown in Fig. 1(b). At $t=300 \tau$, the peak of $\left|\delta n_{i}\right|$ at $x=60 \lambda$ is $0.048 n_{c}$, which is already larger than the threshold value mentioned above, resulting in a local reduction of the growth of SRS. At $t=800 \tau$, the ion density perturbations are developed sufficiently in the whole plasma, and the perturbations exceed the threshold value in most of the plasma region. The linear growth rate of SBS is $\Gamma_{B B S} \approx 0.006$, and the corresponding character time is about $167 \tau$. Considering the width of left vacuum, SBS will develop at $217 \tau$. In our simulation, a strong SBS has been developed in the front of plasma at about $t=280 \tau$. The delay of SBS developments can be attributed to the damping of the incident light due to SRS.

To further demonstrate the SBS effect, we compare two cases with and without ion motion (or infinite ion mass). The lat- 
ter implies that there is no SBS developed. Figure 1(c) shows electron density perturbation spectra associated with the SRS instability. In the case without ion motion, there are two dominant peaks related to SRS at $k_{L}=1.6$ and $k_{L}=0.96$, which indicates intense nonlinear excitation of SRS and SRS rescattering (indicated by the peaks at $k_{L}=0.96$ ). As a comparison, we take a case with $m_{i}=1836$. In this case, SBS is significantly developed as indicated by the peak at $k_{L}=1.92$. In the meanwhile, the peak spectrum of SRS almost disappeared, suggesting that SRS is suppressed by SBS. Due to the fact that SRS can heat electrons ${ }^{9}$, one can use the plasma electron temperature to diagnose the strength of SRS. The average electron temperature is calculated as shown in Fig. 1(d). The plasma heating slows down since $t=700 \tau$ when strong SBS is developed and the subsequent SRS suppression occurs. Note that once the ion density perturbations have grown to certain high level, SRS inhibition can always occur according to our PIC simulations, even though the plasma parameters are no longer in the fluid regime due to significant plasma heating in the early stage.

\section{PIC SIMULATIONS IN INHOMOGENEOUS PLASMA}

\section{A. One-dimensional PIC simulations}

Generally the plasma density is nonuniform in space ${ }^{50}$. For inhomogeneous plasma, the wave number is a function of the space variable. In the linear regime, this problem can be treated by use of the localized equations ${ }^{51}$. Ignoring the low frequency density fluctuation, the SRS instability initially grows exponentially with a growth rate of $\Gamma$, and finally it is saturated because of the phase mismatching ${ }^{52}$. Once the threshold of Eq. (8) is satisfied, the reduction of the SRS saturation level due to SBS induced density perturbations will occur. In the following, we study this nonlinear effect in inhomogeneous plasma by use of PIC simulations.

In this case, the local phase match conditions need to be satisfied, i.e., $\omega_{0}=\omega_{1}+\omega_{L}, k_{0}=k_{1}+k_{L}$. Substituting the dispersion relation of the plasma wave into match conditions for SRS, we have $\omega_{1}=\omega_{0}-\omega_{L}=\omega_{0}-\sqrt{\omega_{p e}^{2}+3 k_{L}^{2} v_{t h}^{2}}$, where $k_{L}=k_{0}+c^{-1} \sqrt{\omega_{0}^{2}-2 \omega_{0} \omega_{p e}}, k_{0}=c^{-1} \sqrt{\omega_{0}^{2}-\omega_{p e}^{2}}$, and $\omega_{p e}(x)$ is a function of $x$. By solving these equations, we can study the instability region using the scattering spectra and wave number distributions. Assuming the initial density distribution is $n_{e}^{0}=0.05[(x-150) / 200+1] n_{c}$. Based on the dispersion relation, we can plot the axis of the instability region varying with the frequency of the back scattering light and the wave number of the electron density as shown in Fig. 2 . Here the frequency and the wave number are normalized to $\omega_{0}$ and $\omega_{0} / c$, respectively. Figure 2(a) indicates that one can easily distinguish the SRS from the SBS according to the scattering spectra. The frequency of SRS ranges from 0.5 to 0.75 and a cutoff is found near $n_{e}^{0}=0.25 n_{c}$. While for SBS, it ranges from 0.99 to 0.993 , indicating a very narrow spectrum around $\omega_{0}$. We can also calculate the instability region from the wave number distributions, as shown in Fig. 2(b). Note
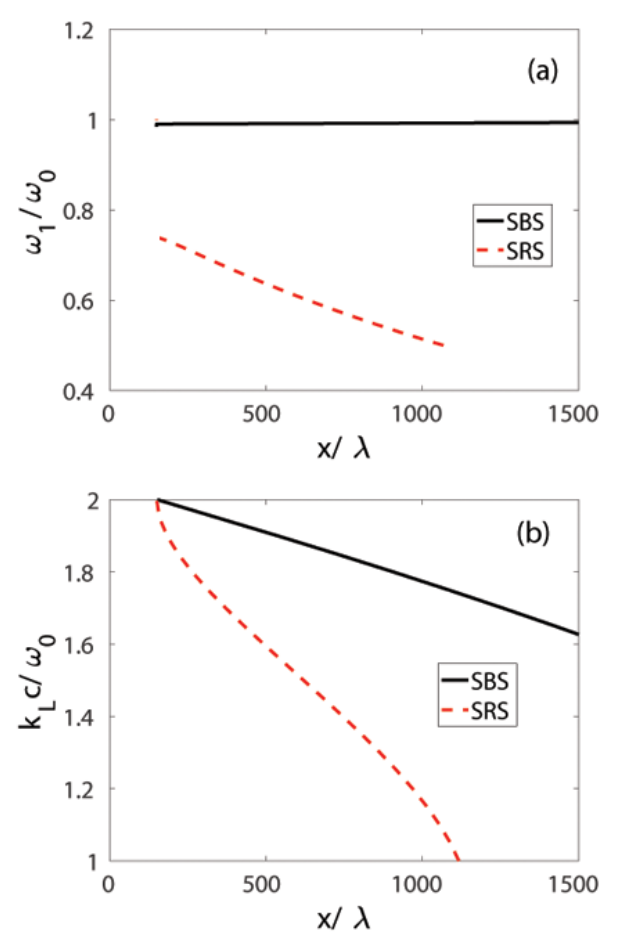

FIG. 2. (a) Changes of the frequencies of the back scattering light via SRS and SBS along the longitudinal coordinate in an inhomogeneous plasma slab adopted in the PIC simulation. (b) The corresponding wave numbers of the Langmuir wave for SRS and the IAW for SBS in the same density profile.

that the wave number of SBS ranges from 1.7 to 2 , which is relatively narrower than SRS. The absolute SRS develops near $n_{e}^{0} \sim 0.25 n_{c}$, where the corresponding $k_{L}$ equals to $k_{0}{ }^{51}$. In this region, SRS is strongest, and the Langmuir and electromagnetic waves can be trapped, producing a large number of hot electrons. These physical pictures are important for us to verify the results of PIC simulations.

In the following 1D simulations, the length of the simulation box is $1500 \lambda$, and the plasma occupies a region from $150 \lambda$ to $1150 \lambda$ with a linear density profile as mentioned above, and initial temperatures $T_{e}=100 \mathrm{eV}$ and $T_{i}=0.5 \mathrm{eV}$, which corresponds to $k_{L} \lambda_{D} \lesssim 0.11$ and $Z T_{e} / T_{i} \gg 10$. To study the evolution of SRS for different strengths of SBS, the ion masses are set to be $m_{i}=1836,5508$, and infinite, respectively. The other parameters are the same as the uniform plasma case above.

Figure 3(a) shows the wave-number spectra of the electron density at different times. At $t=500 \tau$, strong convective SRS has developed and the corresponding $k$ modes are found between $k_{L}=1.5$ and $k_{L}=1.6$. Accordingly one can deduce the instability region is from $x=493 \lambda$ to $x=620 \lambda$ base on Fig. 2(b). At $t=800 \tau$, a small peak near $k_{L}=1.9$ is developed, which corresponds to the SBS spectrum. The spectrum of convective SBS spreads out with increasing time. At $t=1200 \tau$, the instability region is found between $x=345 \lambda$ and $x=1085 \lambda$, and the intensity of the convective SRS is totally suppressed at this time. These results show the evidence 

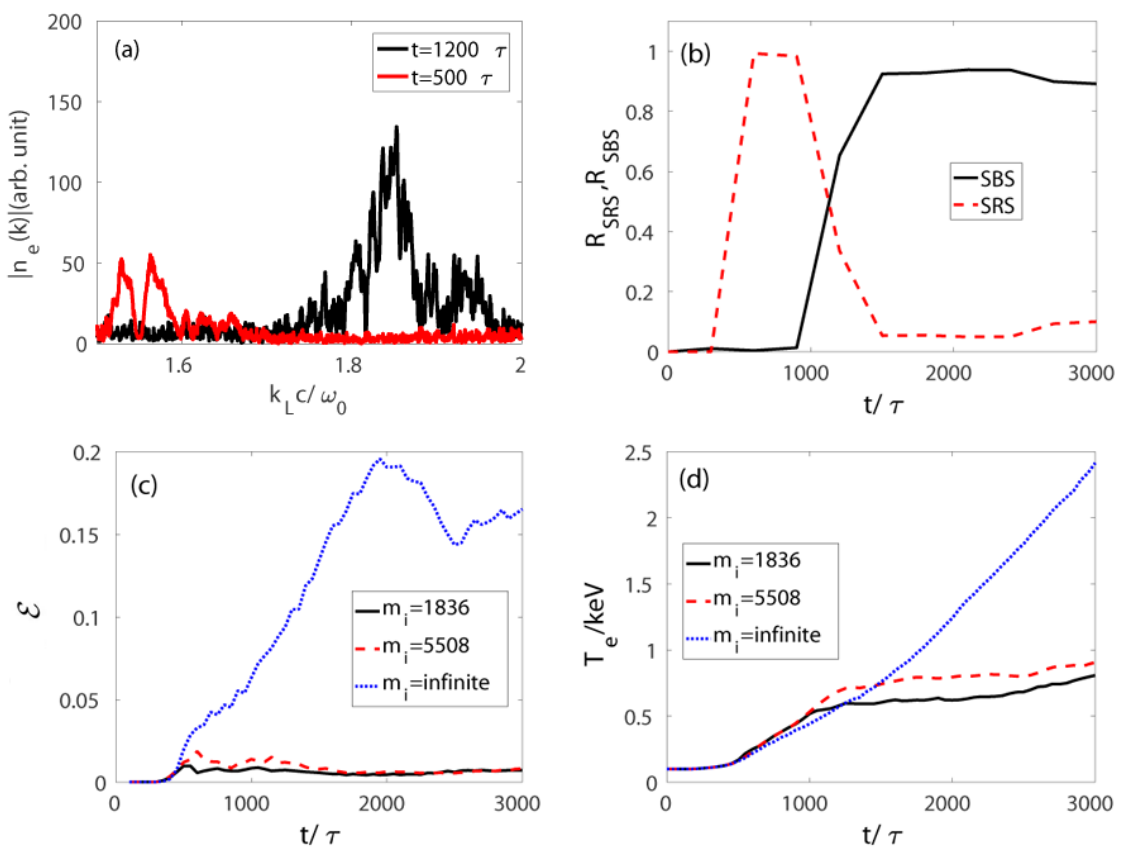

FIG. 3. (a) Electron density spectra in $k$ space at different times with $m_{i}=1836$. (b) Temporal evolution of the ratios of the backscattered SRS and SBS components against the total scattered light with $m_{i}=1836$. The temporal evolutions of the electrostatic energy in plasma (c) and the electron temperature (d) with different ion masses are given to illustrate the effects of SBS excitation on SRS saturation. The laser parameters are the same as in Fig. 1.

of reduced SRS saturation due to the ion density perturbations.

To show more clearly the correlation between SRS and SBS excitation, we define a parameter

$$
R=\int_{\omega_{1}}^{\omega_{2}}\left|E_{z}(\omega)\right|^{2} d \omega / \int_{0}^{\infty}\left|E_{z}(\omega)\right|^{2} d \omega
$$

which defines a ratio between the scattered light energy in certain frequency range and the total scattered light energy. The ratio $R_{S R S}$ for SRS backscattered light is defined with $\left[\omega_{1}, \omega_{2}\right]=[0.5,0.85] \omega_{0}$, while the ratio $R_{S B S}$ for SBS backscattered light is defined with $\left[\omega_{1}, \omega_{2}\right]=[0.95,1.05] \omega_{0}$. The backward lights are diagnosed at $x=50 \lambda$ and the $R$ parameter is calculated per $300 \tau$. Figure 3(b) displays the temporal evolution of ratios of backscattered SRS and SBS light components. It shows that the SRS backscattered light first grows rapidly from $300 \tau$ to $600 \tau$, and then decreases at $t=900 \tau$. Correspondingly, the SBS scattered light grows rapidly from about $t=900 \tau$. This observation clearly illustrates the correlation between the SBS excitation and the SRS suppression.

Another way to measure the level of SRS excitation is to study the evolution of energy of the electrostatic fields stored in the plasma, i.e., $\mathcal{E}=\int\left|E_{x}\right|^{2} d x$. Figure 3(c) shows the temporal evolution of $\mathcal{E}$ under different ion masses. For the case with finite ion mass such as $m_{i}=1836$, it shows that the electrostatic field energy is saturated at $t=700 \tau$. From Fig. 3(a) we know that SBS has been well developed at this time, which helps suppress the further growth of SRS. The saturation time for the case with $m_{i}=5508$ is about $100 \tau$ later due to the lower growth rate of SBS in this case. But the saturation level of $\mathcal{E}$ is very close to the case of $m_{i}=1836$ for their SBS saturation level are similar. If ions are immobile, $\mathcal{E}$ increases linearly with time until $t=2000 \tau$ and finally it is saturated at a much higher level. At $t=3000 \tau, \mathcal{E}$ is found to be $\mathcal{E}=0.165$ which is about twenty times larger than the cases with a finite ion mass $m_{i}=1836$ or $m_{i}=5508$.

The change of plasma electron temperatures is closely related to the SRS development. Figure 3(d) shows clearly the reduced electron heating when the SRS is suppressed due to the SBS excitation. In the early stage from $400 \tau$ to $900 \tau$, the plasma electron temperature increases for all three cases with different ion masses. After $1000 \tau$, for the case with $m_{i}=1836$, the temperature grows slowly and tends to saturate around 0.9 $\mathrm{keV}$. At this time, the percent SBS is $R_{S B S} \sim 10 \%$, which indicates that strong SBS has been developed. For $m_{i}=5508$, the saturation time is around $1250 \tau$, which is slower than the case of $m_{i}=1836$ due to the smaller growth rate of SBS. The saturation level of the heating is slightly higher than the case of $m_{i}=1836$. Note that the saturation level of SRS in inhomogeneous plasma always smaller than the homogeneous case, due to its density gradient. For the case with immobile ions, the temperature grows almost linearly with time, and has no saturation trend. At $t=3000 \tau$, the electron temperature is $T_{e} \sim 2.5 \mathrm{keV}$, which is almost three times larger than the case of $m_{i}=1836$. The above comparison indicates that the development of SBS can lead to a significant suppression of SRS and consequent electron heating. 

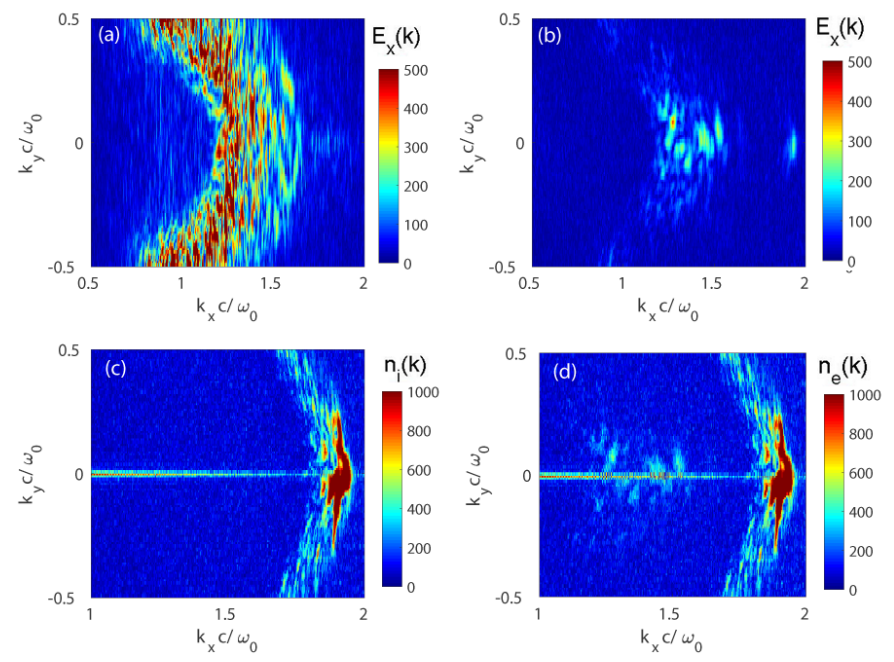

FIG. 4. (a) and (b) Wave number distributions of the longitudinal electric field at $t=700 \tau$ with immobile ions and $m_{i}=1836$, respectively. (c) and (d) Wave number distributions of the ion and electron density perturbations with $m_{i}=1836$ at $t=700 \tau$, respectively.

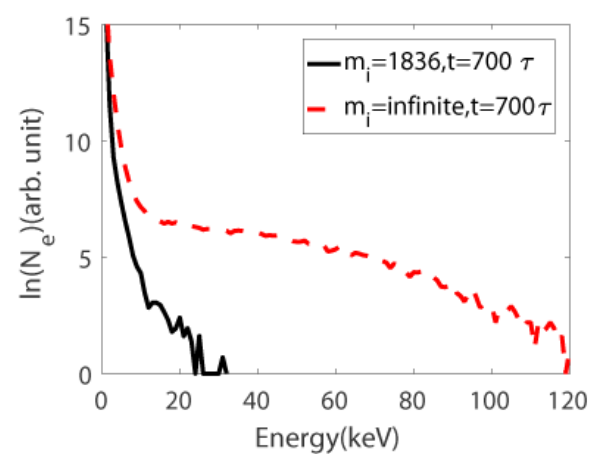

FIG. 5. Energy spectra of electrons with different ion masses at $t=$ $700 \tau$.

\section{B. Two-dimensional PIC simulations}

To study the SBS suppression effects on SRS and plasma heating in multi-dimensional laser plasma interactions, we also have performed two-dimensional (2D) PIC simulations. The length and width of our simulation box are $600 \lambda$ and $100 \lambda$, respectively. Plasma occupies a region from $x=50 \lambda$ to $x=550 \lambda$ with a linear density profile $n_{e}^{0}(x)=0.05[(x-$ $50) / 150+1] n_{c}$, and uniformly distributes in the transverse direction. The initial temperatures are $T_{e}=100 \mathrm{eV}$ and $T_{i}=0.5$ $\mathrm{eV}$. We take 40 cells and 20 cells per wavelength respectively in longitudinal and transverse directions with 2 particles per cell. A laser normally incidents en the plasma with Gaussian distribution in the transverse direction, its electric field is perpendicular to the simulation plane with amplitude $a_{0}=0.05$. The radius of the focal spot is $8 \lambda$, and its center is located at $y=50 \lambda$. We use the reflection boundary conditions for particles in two directions.
As discussed above, the convective SRS instability develop$\mathrm{s}$ in the low-density region, and then spreads out to the highdensity region. In our simulations, the longitudinal wavenumber of the Langmuir wave for convective SRS and SBS are found at $k_{x}<1.72$ and $k_{x} \sim 1.9$, respectively. For immobile ions, strong convective SRS instabilities developed in the whole plasma at $t=700 \tau$, which is shown in Fig. 4(a). As a comparison, the strength of convective SRS is much smaller with $m_{i}=1836$, as shown in Fig. 4(b). A strong mode for SBS can be found near $k_{x} \simeq 1.93$. The wave number distribution of ion density perturbations at $t=700 \tau$ is plotted in Fig. 4(c), where a strong mode near $k_{x} \simeq 1.93$ due to SBS is found. Figure 4(d) shows that the SRS mode has been suppressed considerably when the SBS mode $k_{x} \simeq 1.93$ plays a dominant role. The electron heating for immobile ions is much more significantly than the case with $m_{i}=1836$ at $t=700 \tau$, as shown in Fig. 5. All these results are in good agreement with $1 \mathrm{D}$ simulation results.

\section{SUMMARY}

In this work we have investigated the effects of SBS excitation on the inhibition of SRS. A set of coupling equations for SRS and SBS are given, describing the four wave interactions under different conditions. Without ion density perturbations, these equations reduced to the equations for the pure SRS instability. The SRS instability in uniform plasma with an ion density perturbation is analyzed. A threshold perturbation for the saturation of the SRS instability is derived, which is confirmed qualitatively by 1D PIC simulations in uniform plasma. Our simulation results indicate that in the fluid regime of the SRS development with $k_{L} \lambda_{D} \lesssim 0.15$, the threshold condition of ion density perturbations for SRS saturation can be satisfied when SBS develops. Once SBS has developed to a certain high level, its suppression effects on SRS still holds even if the fluid regime condition is violated due to plasma heating.

The suppression on SRS due to SBS is also found in inhomogeneous plasma. Both 1D and 2D PIC simulations in inhomogeneous plasma have shown explicitly the correlation of the Langmuir wave and SBS. The latter can significantly suppress SRS and subsequent plasma electron heating by exciting large amplitude ion density perturbations.

\section{ACKNOWLEDGMENTS}

This work was supported in part by the National Science Foundation of China (Grant Nos. 11421064, 11129503, 11374209,11405107 and 11374210). C.R. is also supported by U.S. DOE under Grant Nos. DE-FC02-04ER54789 and DE-SC0012316 and by NSF under Grant No. PHY1314734. Simulations have been carried out on the PI supercomputer at Shanghai Jiao Tong University and the Milky Way 2 supercomputer in the National Supercomputer Center in Guangzhou. 
${ }^{1}$ S. Glenzer, P. Arnold, G. Bardsley, R. Berger, G. Bonanno, T. Borger, D. Bower, M. Bowers, R. Bryant, S. Buckman, et al., Nucl. Fusion 44, S185 (2004).

${ }^{2}$ O. Klimo, S. Weber, V. T. Tikhonchuk, and J. Limpouch, Plasma Phys. Control. Fusion 52, 055013.

${ }^{3}$ J. Lindl, Phys. Plasmas 2, 3933 (1995)

${ }^{4}$ S. Glenzer, B. MacGowan, P. Michel, N. Meezan, L. Suter, S. Dixit, J. Kline, G. Kyrala, D. Bradley, D. Callahan, et al., Science 327, 1228 (2010).

${ }^{5}$ B. Winjum, J. Fahlen, F. Tsung, and W. Mori, Phys. Rev. Lett. 110, 165001 (2013).

${ }^{6}$ S. Brunner and E. Valeo, Phys. Rev. Lett. 93, 145003 (2004).

${ }^{7}$ H. X. Vu, D. DuBois, and B. Bezzerides, Phys. Rev. Lett. 86, 4306 (2001).

${ }^{8}$ P. E. Masson-Laborde, W. Rozmus, Z. Peng, D. Pesme, S. Hüller,

M. Casanova, V. Y. Bychenkov, T. Chapman, and P. Loiseau, Phys. Plasmas 17, 092704 (2010).

${ }^{9}$ W. L. Kruer, The physics of laser plasma interactions, Vol. 70 (AddisonWesley New York, 1988).

${ }^{10}$ B. Winjum, J. Fahlen, and W. Mori, Phys. Plasmas 14, 102104 (2007).

${ }^{11}$ B. J. Winjum, R. L. Berger, T. Chapman, J. W. Banks, and S. Brunner, Phys. Rev. Lett. 111, 105002 (2013).

${ }^{12}$ L. Yin, B. J. Albright, K. J. Bowers, W. Daughton, and H. A. Rose, Phys. Rev. Lett. 99, 265004 (2007).

${ }^{13}$ J. W. Banks, R. L. Berger, S. Brunner, B. I. Cohen, and J. A. F. Hittinger, .

${ }^{14}$ J. Kline, D. Montgomery, L. Yin, D. DuBois, B. Albright, B. Bezzerides, J. Cobble, E. Dodd, J. Fernández, and R. Johnson, Phys. Plasmas 13, 055906 (2006).

${ }^{15}$ S. Hüller, P. E. Masson-Laborde, D. Pesme, M. Casanova, F. Detering, and A. Maximov, Phys. Plasmas 13, 022703 (2006).

${ }^{16}$ R. E. Giacone and H. X. Vu, Phys. Plasmas 5, 1455 (1998).

${ }^{17}$ T. Chapman, B. J. Winjum, S. Brunner, R. L. Berger, and J. W. Banks, Phys. Plasmas 22, 092116 (2015).

${ }^{18}$ J. J. Thomson and J. I. Karush, Phys. Fluids 17, 1608 (1974).

${ }^{19}$ Y. Zhao, L.-L. Yu, J. Zheng, S.-M. Weng, C. Ren, C.-S. Liu, and Z.-M. Sheng, Phys. Plasmas 22, 052119 (2015).

${ }^{20}$ Y. Zhao, J. Zheng, L.-L. Yu, M. Chen, S.-M. Weng, and Z.-M. Sheng, Sci China G-Phys Mech Astron 45, 035201 (2015).

${ }^{21}$ P. Loiseau, O. Morice, D. Teychenné, M. Casanova, S. Hüller, and D. Pesme, Phys. Rev. Lett. 97, 205001 (2006).

${ }^{22}$ S. Skupsky, R. W. Short, T. Kessler, R. S. Craxton, S. Letzring, and J. M. Soures, J. Appl. Phys. 66, 3456 (1989).

${ }^{23}$ S. P. Regan, J. A. Marozas, J. H. Kelly, T. R. Boehly, W. R. Donaldson, P. A. Jaanimagi, R. L. Keck, T. J. Kessler, D. D. Meyerhofer, W. Seka, S. Skupsky, and V. A. Smalyuk, J. Opt. Soc. Am. B 17, 1483 (2000).

${ }^{24}$ R. H. Lehmberg and S. P. Obenschain, Optics Comm. 46, 27 (1983).

${ }^{25}$ D. H. Froula, L. Divol, R. A. London, R. L. Berger, T. Döppner, N. B.
Meezan, J. Ralph, J. S. Ross, L. J. Suter, and S. H. Glenzer, Phys. Plasmas 17, 056302 (2010).

${ }^{26}$ J. Fuchs, C. Labaune, S. Depierreux, H. A. Baldis, and A. Michard, Phys. Rev. Lett. 84, 3089 (2000).

${ }^{27}$ J. D. Moody, B. J. MacGowan, J. E. Rothenberg, R. L. Berger, L. Divol, S. H. Glenzer, R. K. Kirkwood, E. A. Williams, and P. E. Young, Phys. Rev. Lett. 86, 2810 (2001).

${ }^{28}$ Y. Zhao, J. Zheng, M. Chen, L.-L. Yu, S.-M. Weng, C. Ren, C.-S. Liu, and Z.-M. Sheng, Phys. Plasmas 21, 112114 (2014).

${ }^{29}$ V. E. Zakharov, Sov. Phys. JETP 35, 908 (1972).

${ }^{30}$ G. Doolen, D. DuBois, and H. A. Rose, Phys. Rev. Lett. 54, 804 (1985).

${ }^{31}$ E. Kuznetsov, A. Rubenchik, and V. Zakharov, Phys. Reports 142, 103 (1986).

${ }^{32}$ B. Bezzerides, D. DuBois, and H. A. Rose, Phys. Rev. Lett. 70, 2569 (1993).

${ }^{33}$ R. Sharma, K. Batra, and A. Verga, Phys. Plasmas 12, 022311 (2005).

${ }^{34}$ X. T. He and C. Y. Zheng, Phys. Rev. Lett. 74, 78 (1995).

${ }^{35}$ S. J. Karttunen, Phys. Rev. A 23, 2006 (1981).

${ }^{36}$ G. Bonnaud, D. Pesme, and R. Pellat, Phys. Fluids B 2, 1618 (1990).

${ }^{37}$ L. Hao, R. Yan, J. Li, W. D. Liu, and C. Ren, Phys. Plasmas 24, 565 (2017).

${ }^{38}$ C. Walsh, D. Villeneuve, and H. Baldis, Phys. Rev. Lett. 53, 1445 (1984).

${ }^{39}$ D. Villeneuve, H. Baldis, and J. Bernard, Phys. Rev. Lett. 59, 1585 (1987).

${ }^{40}$ H. Baldis, D. Villeneuve, C. Labaune, D. Pesme, W. Rozmus, W. Kruer, and P. Young, Phys. Fluids B 3, 2341 (1991).

${ }^{41}$ C. Labaune, H. Baldis, N. Renard, E. Schifano, and A. Michard, Phys. Plasmas 4, 423 (1997).

${ }^{42}$ T. D. Chapman, Autoresonance in stimulated Raman scattering, Ph.D. thesis, Citeseer (2012).

${ }^{43}$ D. Forslund, J. Kindel, and E. Lindman, Phys. Fluids 18, 1002 (1975).

${ }^{44}$ S. Karttunen, Plasma Physics 22, 151 (1980).

${ }^{45}$ W. Rozmus, R. Sharma, J. Samson, and W. Tighe, Phys. Fluids 30, 2181 (1987).

${ }^{46}$ C. Riconda, A. Heron, D. Pesme, S. Hüller, V. T. Tikhonchuk, and F. Detering, Phys. Rev. Lett. 94, 055003 (2005).

${ }^{47}$ S. Weber, C. Riconda, and V. T. Tikhonchuk, Phys. Rev. Lett. 94, 055005 (2005).

${ }^{48}$ B. I. Cohen, L. Divol, A. B. Langdon, and E. A. Williams, Phys. Plasmas 12, 052703 (2005).

${ }^{49}$ M. Chen, Z.-M. Sheng, J. Zheng, Y.-Y. Ma, and J. Zhang, Chin. J. Comput. Phys. 25, 43 (2008)

${ }^{50}$ J. Myatt, J. Zhang, R. Short, A. Maximov, W. Seka, D. Froula, D. Edgell, D. Michel, I. Igumenshchev, D. Hinkel, et al., Phys. Plasmas 21, 055501 (2014).

${ }^{51}$ C. Liu, M. N. Rosenbluth, and R. B. White, Phys. Fluids 17, 1211 (1974).

${ }^{52}$ M. Rosenbluth, R. White, and C. Liu, Physical Review Letters 31, 1190 (1973). 